\title{
Volumetric PET Response Assessment Outperforms Conventional Criteria in Patients Receiving High-Dose Pembrolizumab for Malignant Mesothelioma
}

\author{
Justin Ferdinandus ${ }^{1}$, Francesco Barbato ${ }^{1}$, Michal Chodyla ${ }^{2}$, Wolfgang P. Fendler ${ }^{1}$, Lukas Kessler ${ }^{1}$, Kelsey L. Pomykala $^{1,3}$, \\ Martin Metzenmacher ${ }^{4}$, Frederik Krefting ${ }^{5}$, Thomas Hager ${ }^{6}$, Lale Umutlu ${ }^{3}$, Ken Herrmann ${ }^{1}$, and Daniel C. Christoph ${ }^{4}$ \\ ${ }^{1}$ Department of Nuclear Medicine, University of Duisburg-Essen, and German Cancer Consortium-University Hospital Essen, Essen, \\ Germany; ${ }^{2}$ Department of Diagnostic and Interventional Radiology and Neuroradiology, University Hospital Essen, University of \\ Duisburg-Essen, Essen, Germany; ${ }^{3}$ Department of Radiology, UCLA, Los Angeles, California; ${ }^{4}$ Department of Medical Oncology, \\ University Hospital Essen, University of Duisburg-Essen, Essen, Germany; ${ }^{5}$ Department of Dermatology, University Hospital Essen, \\ University of Duisburg-Essen, Essen, Germany; and ${ }^{6}$ Institute of Pathology, University Hospital Essen, University of Duisburg- \\ Essen, Essen, Germany
}

Fixed-dose pembrolizumab (200 mg absolute, day 1 , every $3 \mathrm{wk}$ ) for the treatment of malignant pleural mesothelioma did not result in survival benefit in the phase 3 PROMISE-meso trial compared with second-line chemotherapy. Because of lack of validated imaging response criteria, responder subgroups with potential survival benefit have not yet been identified. Here, we administered highdose pembrolizumab (10 mg/kg, day 1 , every $2 \mathrm{wk}$ ) considering the KEYNOTE-028 trial and assessed the prognostic value of PET metabolic response in patients with chemotherapy-resistant malignant mesothelioma of the pleura or peritoneum. Methods: Data from 27 patients with baseline and follow-up ${ }^{18} \mathrm{~F}-\mathrm{FDG}$ PET/CT imaging were retrospectively analyzed. RECIST, version 1.1; modified RECIST; and PERCIST using both tumor lesion metabolic activity in a $1 \mathrm{~cm}^{3}$ spheric region of interest of up to 5 target lesions (PERCIST SULpeak $_{\text {) and metabolic tumor volume PERCIST (PERCIST }}$ MTV) were applied separately to categorize responders in CT and PET imaging studies. Progression-free survival (PFS) and overall survival (OS) were compared between responders and nonresponders using Kaplan-Meier and log-rank analyses. Programmed cell death protein 1 ligand expression status was assessed, and its association with outcome was investigated. Results: Twenty-seven patients had ${ }^{18}$ F-FDG PET/CT imaging at baseline and after at least 4 cycles pembrolizumab. Median PFS and OS were 3.4 and $15.1 \mathrm{mo}$, respectively. Response rates were $7 \%, 7 \%, 30 \%$, and $30 \%$ based on RECIST, modified RECIST, PERCIST SULpeak, and PERCIST $_{\text {MTV re- }}$ sponse criteria, respectively. Response according to PERCIST MTV $_{\text {. }}$ predicted prolonged OS or PFS $(P<0.01)$, whereas all other imaging criteria and programmed cell death protein 1 ligand expression did not. Conclusion: ${ }^{18}$ F-FDG PET metabolic volume response predicts survival in patients with malignant mesothelioma receiving high-dose pembrolizumab. These results should prompt inclusion of PET response assessment in future phase 3 clinical trials.

Received Mar. 24, 2020; revision accepted May 19, 2020.

For correspondence or reprints contact: Justin Ferdinandus, University Hospital Essen, Department of Nuclear Medicine, Hufelandstrasse 55, 45147, Essen, Germany.

E-mail: justin.ferdinandus@uk-essen.de

Published online Jun. 12, 2020.

COPYRIGHT (c) 2021 by the Society of Nuclear Medicine and Molecular Imaging.
Key Words: mesothelioma; checkpoint inhibitor; pembrolizumab; $\mathrm{PET} / \mathrm{CT}$

J Nucl Med 2021; 62:191-194

DOI: 10.2967/jnumed.120.245803

$\mathbf{P}$ atients with malignant mesothelioma of the pleura have a poor prognosis, with a median overall survival (OS) of between 12 and 18 mo and a 5-y survival rate of less than 5\% (1). Patients with peritoneal mesothelioma have an even more dismal prognosis (2). On progression after first-line chemotherapy, therapeutic options are limited and have moderate efficacy $(3,4)$. The KEYNOTE-028 phase Ib trial of pembrolizumab revealed high disease control rates as evidenced by patient CT scans and a median OS of 18 mo in patients for whom standard therapies fail (5). Of note, a subset of these patients achieved durable responses after programmed cell death protein 1 inhibition. However, only RECIST was applied to assess imaging response. Here, we compare currently available response criteria for pembrolizumab treatment in malignant mesothelioma in a real-world setting, including PET-based response assessment. Our aim was early identification of patients who achieve a durable response to high-dose pembrolizumab, justifying this costly treatment regimen and avoiding further costs and adverse events in nonresponders.

\section{MATERIALS AND METHODS}

This was a single-arm retrospective study of patients receiving high-dose pembrolizumab (10 mg/kg, day 1 , every $2 \mathrm{wk})$ off-label for a maximum of $2 \mathrm{y}$ after approved by each patient's health insurance company (Supplemental Fig. 1; supplemental materials are available at http://jnm.snmjournals.org). After pembrolizumab became commercially available in June 2015, applications for use were submitted for every patient without autoimmune disease whose disease progressed after at least 1 line of platinum- and pemetrexed-based combination chemotherapy. The inclusion criteria for the study required a PET/CT examination at baseline and after 4 cycles, as per clinical routine for offlabel use of expensive drugs. One patient received his scan after 5 cycles for logistic reasons. Applications for high-dose pembrolizumab were stopped after approval of the fixed-dose regimen in March 2017. Patients 
received high-dose pembrolizumab from June 2015 to December 2017. Follow-up was finished in December 2019. The institutional review board (or equivalent) approved this study, and all subjects gave written informed consent.

\section{Image Acquisition and Analysis}

Dual-modality PET/CT was performed on a Siemens Biograph mCT or a Siemens Biograph Duo system. Patients received a mean dose of $260 \mathrm{MBq}$ of ${ }^{18} \mathrm{~F}-\mathrm{FDG}$ (range, 206-343 MBq) and were scanned after a mean of $65 \mathrm{~min}$ after injection (range, 49-105 min). The CT images were used for PET attenuation correction. Diagnostic CT scans with intravenous and oral contrast agents were performed, with the exception of 2 patients with reduced kidney function. Follow-up imaging was performed on the same scanner as was used for baseline imaging.

On image analysis, readers were masked to the outcomes of the patients. Morphologic response from baseline and follow-up PET/CT was analyzed by an experienced radiologist using RECIST, version 1.1, and revised modified RECIST for Assessment of Response in Malignant Pleural Mesothelioma, version 1.1 (modified RECIST, or mRECIST) (6). Metabolic response was analyzed using PERCIST in 2 ways: the first was with tumor lesion metabolic activity in a $1 \mathrm{~cm}^{3}$ spheric region of interest of up to 5 target lesions (PERCIST SULpeak $_{\text {), }}$ and the second was with whole-body metabolic tumor volume (MTV), defined as the volume of all lesions with uptake above 1.5 times the mean uptake in liver plus 2 SDs of uptake in the liver (PERCIST ${ }_{\text {MTV }}$ ). An MTV of $0 \mathrm{~cm}^{3}$ in the follow-up scan was classified as complete response. A decline in MTV by at least 30\% was classified as partial response, and an increase by at least $30 \%$ was classified as progressive disease. New lesions were automatically encompassed by MTV measurements (given that they were above the evaluation threshold) and were not automatically considered progression, as long as there was no MTV increase of $30 \%$ or more (7).

\section{Programmed Cell Death Protein 1 Ligand (PD-L1) \\ Expression Profile}

Formalin-fixed and paraffin-embedded tissue samples were obtained for routine diagnostics and stained in standard hematoxylin and eosin. Dried paraffin slides were generated from the tissue blocks. Further steps were performed with a Ventana Benchmark Ultra automated immune histochemical slide staining system using the antibody clone 28-8 (Abcam). Immunostainings were evaluated by a pathologist, whose experience was confirmed in round-robin tests, by scanning each slide under low-power $(\times 20)$ and high-power $(\times 200)$ magnification. PD-L1-positive tumor cells were identified by continuous membrane staining. Positive tumor cells were quantified as percentage per slide (positive tumor rates with reference to the whole tumor on the slide).

Adverse events were recorded and graded using the Common Terminology Criteria for Adverse Events, version 4.0.

\section{Statistical Analysis}

OS and progression-free survival (PFS) of response groups and PDL1 expression profiles were compared using Kaplan-Meier methods and log-rank testing. $P$ values lower than 0.05 were regarded as statistically significant. Statistical analysis was performed using R statistics (version 3.4.1, www.r-project.org).

\section{RESULTS}

Fifty-two patients with malignant mesothelioma experienced progression at least after first-line chemotherapy and were deemed eligible for pembrolizumab treatment (Supplemental Fig. 1). Because of denied applications from health insurance companies or death before or shortly after the baseline PET/CT examination, 37 patients started pembrolizumab treatment after baseline PET/CT.
TABLE 1

Patient Characteristics $(n=27)$

\begin{tabular}{lc}
\hline \multicolumn{1}{c}{ Characteristic } & Data \\
\hline Sex & $23(85.2 \%)$ \\
\hline Male & $4(14.8 \%)$ \\
\hline Female & $68($ range, $51-82)$ \\
\hline Median age at diagnosis (y) & \\
\hline IMIG/IASLC stage (if applicable) & $3(11.1 \%)$ \\
\hline 2 & $8(29.6 \%)$ \\
\hline 3 & $16(59.3 \%)$ \\
\hline 4 & \\
\hline Histologic subtype & $21(77.8 \%)$ \\
\hline Epitheloid & $3(11.1 \%)$ \\
\hline Sarcomatoid & $2(7.4 \%)$ \\
\hline Biphasic & $1(3.7 \%)$ \\
\hline Desmoplastic & \\
\hline Prior lines of chemotherapy & $13(48.1 \%)$ \\
\hline 2 & $8(29.6 \%)$ \\
\hline 3 & $6(22.2 \%)$ \\
\hline$\geq 4$ & $12(44.4 \%)$ \\
\hline PD-L1 expression & $11(40.7 \%)$ \\
\hline $0 \%$ & $3(11.1 \%)$ \\
\hline $1 \%-49 \%$ & $1(3.7 \%)$ \\
\hline $50 \%-100 \%$ & \\
\hline Missing & \\
\hline
\end{tabular}

IMIG/IASLC = International Mesothelioma Interest Group/International Association for the Study of Lung Cancer.

Data are $n$ followed by percentage in parentheses, except for age.

However, because of clinical deterioration and early abruption of treatment, only 27 patients underwent baseline and follow-up PET/ CT after 4 cycles and were evaluable for this analysis. The patient characteristics are summarized in Table 1.

Median follow-up was 29.7 mo. Median OS and PFS were 15.1 and 3.4 mo, respectively (Fig. 1). Treatment with high-dose pembrolizumab was well tolerated (Supplemental Table 1).

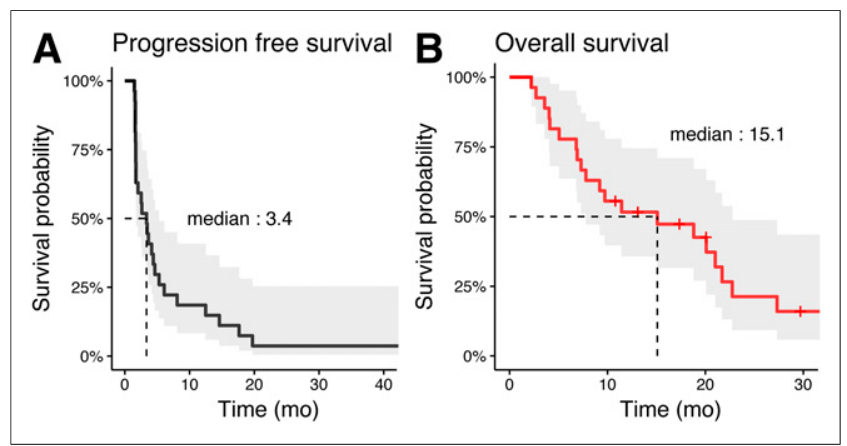

FIGURE 1. PFS (A) and OS (B) in 27 mesothelioma patients who were treated with pembrolizumab and underwent baseline and follow-up PET imaging. Median PFS was 3.4 mo; median OS was 15.1 mo. 
TABLE 2

Response Rates of PET/CT Imaging Using Different Response Criteria

\begin{tabular}{lcllrr}
\hline \multicolumn{1}{c}{ Criteria } & Objective response & CR/CMR & PR/PMR & SD/SMD & PD/PMD \\
\hline RECIST $^{*}$ & $2(7.4 \%)$ & $0(0 \%)$ & $2(7.4 \%)$ & $12(44.4 \%)$ & $12(44.4 \%)$ \\
mRECIST* $^{*}$ & $2(7.4 \%)$ & $0(0 \%)$ & $2(7.4 \%)$ & $11(40.7 \%)$ & $13(48.1 \%)$ \\
PERCIST $_{\text {SULpeak }}$ & $8(29.6 \%)$ & $1(3.7 \%)$ & $7(25.9 \%)$ & $10(37.0 \%)$ & $9(33.3 \%)$ \\
PERCIST $_{\text {MTV }}$ & $8(29.6 \%)$ & $0(0 \%)$ & $8(29.6 \%)$ & $6(22.2 \%)$ & $13(48.1 \%)$ \\
*1 patient with no measurable target lesion at baseline. & & & & \\
Data are $n$ followed by percentage in parentheses. & & & & \\
\hline
\end{tabular}

All but 1 patient died because of malignant disease. The objective response rates of RECIST, mRECIST, PERCIST SULpeak $_{\text {, }}$

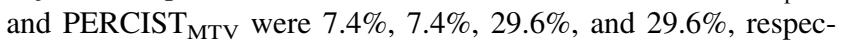
tively. Table 2 demonstrates response groups identified by each set of criteria. RECIST and mRECIST were concordant in 23 of 27

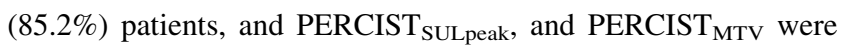
concordant in 14 of $27(51.2 \%)$ patients. Only response identified

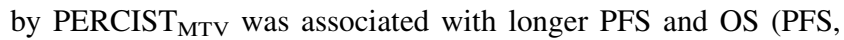
median of 1.7 vs. $13.5 \mathrm{mo}$; OS, median of 9.2 vs. $24.5 \mathrm{mo}$; both $P<0.01$ ). Kaplan-Meyer curves presenting survival stratified by imaging response are presented in Figure 2 and Supplemental Figure 2. PD-L1 expression was available for 26 of 27 (96.3\%) patients; approximately half the patients had tumors with a lack of PD-L1 expression. Expression profiles could not be associated with response by any imaging criteria (Supplemental Table 2). There was no significant difference in OS or PFS between patients whose tumors express PD-L1 and those whose tumors did not (Supplemental Fig. 3).

\section{DISCUSSION}

Malignant mesothelioma remains a disease with limited treatment options. For patients with locally advanced or metastatic disease, combination chemotherapy consisting of a platinum compound and pemetrexed is the standard of care (8). Currently, no second-line standard of care exists. Therefore, experimental treatment with programmed cell death protein 1 inhibitors such as pembrolizumab instead of single-agent chemotherapy with gemcitabine or vinorelbine seems to be justified, if health insurance companies are willing to support this proposal and reimburse the high expenses of currently available checkpoint inhibitors.

Although ${ }^{18} \mathrm{~F}$-FDG PET/CT has been studied for the assessment of response to therapy in mesothelioma, the use of this imaging modality in response assessment and as a surrogate for patient benefit in clinical trials has not been validated (6). At present, ${ }^{18} \mathrm{~F}$-FDG PET is not a validated tool for the assessment of treatment response in clinical trials.

In this retrospective study, we demonstrated that an early change in PET metabolic volume identifies patient responders to pembrolizumab. This finding is of particular relevance, as both RECIST and mRECIST, commonly used in clinical trials, have not been able to predict survival in this setting. Potentially, RECIST fails to identify partial responders in the assessment of nonspheroid disease, which is a general problem in mesothelioma. Especially in immunotherapy, the morphology or thickness as assessed by mRECIST might not be the best marker of response, as an early increase in thickness after T-cell infiltration and inflammatory response might occur. Furthermore, PET is noninvasive and allows rapid and reproducible quantification of disease - a capability that is of specific use in mesothelioma, given the rather sheetlike, nonspheroid growth of these tumors. Early detection of responding patients can identify those for whom expensive continuation of high-dose pembrolizumab results in clinically relevant, improved survival and is justified.

Low, fixed-dose pembrolizumab (200 mg absolute, day 1 , every $3 \mathrm{wk}$ ) for the treatment
FIGURE 2. OS stratified by responders (blue) and nonresponders (red) identified by different response assessment criteria. Log-rank test revealed that only PERCIST ${ }_{\text {MTV }}$ responders (D) showed significantly longer survival than nonresponders (median, 9.2 vs. 24.5 mo). 
of malignant pleural mesothelioma did not result in survival benefit in the phase 3 PROMISE-meso trial compared with second-line chemotherapy (9). In contrast, in phase $1 \mathrm{~b}$ trials investigating high-dose pembrolizumab in 25 patients with malignant pleural mesothelioma whose tumors had to express PD-L1, PFS was 5.4 and OS was 18 mo (5). In our study, less than half the patients had a tumor with PD-L1 expression and received pembrolizumab as second-line treatment, and no association between PD-L1 expression and response was observed. A recently published retrospective study investigating different dosing schemes for pembrolizumab (mainly a low, fixed dose of pembrolizumab) in 93 patients with malignant pleural mesothelioma as second- and further-line treatment demonstrated rather low median PFS and OS times (3.1 and 7.2 mo, respectively) (10). The heterogeneity of currently available evidence highlights the need for an accurate early-response biomarker to identify individual patients benefitting from immunotherapy.

To the best of our knowledge, no other prospective study has been published on the value of PET/CT diagnostics in predicting response and improved survival due to PD1-antibody treatment in mesothelioma patients. A decline in ${ }^{18} \mathrm{~F}-\mathrm{FDG}$ PET/CT MTV or total-lesion glycolysis is associated with longer survival in mesothelioma patients undergoing pemetrexed-based chemotherapy $(11,12)$. Here, we find a similar association in patients undergoing immunotherapy.

Although our study benefited from a homogeneously treated cohort and long-term follow-up (all but one of the patients died), it had a few limitations. Primarily, our sample size did not allow us to draw definitive conclusions on whether PET in general, and MTV response assessment specifically, are able to guide therapeutic management in this setting. Second, patients with malignant mesothelioma of the pleura or the peritoneum were included; the latter is associated with a dramatically poorer prognosis (2). Finally, patients who succumbed to their disease before follow-up PET/CT after 4 cycles could not be considered in the efficacy analysis.

\section{CONCLUSION}

We demonstrated that PERCIST-derived metabolic volume response predicts survival in pretreated patients with malignant mesothelioma receiving high-dose pembrolizumab. No other imaging criteria predicted survival. The results warrant inclusion of PET response assessment in future clinical trials and routine practice.

\section{DISCLOSURE}

Justin Ferdinandus received a Junior Clinician Scientist Stipend from the University Duisburg-Essen. Wolfgang Fendler is a consultant for Ipsen, Endocyte, and BTG and received personal fees from RadioMedix, outside the submitted work. Lukas Kessler receives personal fees from Sanofi, outside the submitted work. Martin Metzenmacher receives personal fees from BoehringerIngelheim, Roche, Takeda, Bristol-Myers Squibb, and Merck, Sharp \& Dohme, outside the submitted work. Thomas Hager receives personal fees from Merck, Sharp \& Dohme, Bristol Myers Squibb, Roche Pharma, and Chugai Pharma, outside the submitted work. Ken Herrmann receives personal fees from Bayer, Sofie Biosciences, SIRTEX, Curium, Endocyte, BTG, IPSEN, Siemens Healthineers, GE Healthcare, Amgen, Novartis, and ymabs; grants from BTG; nonfinancial support from $\mathrm{ABX}$; and other fees from Sofie Biosciences, outside the submitted work. Daniel Christoph receives personal fees, nonfinancial support and other fees from AstraZeneca, Bayer, Boehringer-Ingelheim, Bristol-Myers Squibb, Chugai, Merck, Sharp \& Dohme, Novartis, Pfizer, Roche, and
Takeda, outside the submitted work. No other potential conflict of interest relevant to this article was reported.

\section{ACKNOWLEDGMENTS}

We thank diverse health insurance companies for approving high-dose pembrolizumab treatment on an individual basis with the condition of performing PET/CT diagnostics at baseline and during treatment for early efficacy evaluation of patients with pretreated malignant mesothelioma.

\section{KEY POINTS}

QUESTION: Is response to immunotherapy in mesothelioma measurable, and does it predict survival?

PERTINENT FINDINGS: We performed a retrospective study and tested whether tumor response classified by RECIST, mRECIST, PERCIST $_{\text {sULpeak, }}$, or PERCIST MTV $_{\text {was associated with improved }}$ OS. Only volumetric response was associated with longer OS.

IMPLICATIONS FOR PATIENT CARE: Volumetric PET response criteria should be implemented in future clinical trials and are useful for monitoring patients receiving off-label immunotherapy after first-line treatments have failed.

\section{REFERENCES}

1. Mutti L, Peikert T, Robinson BWS, et al. Scientific advances and new frontiers in mesothelioma therapeutics. J Thorac Oncol. 2018;13:1269-1283.

2. Turner K, Varghese S, Alexander HR Jr. Current concepts in the evaluation and treatment of patients with diffuse malignant peritoneal mesothelioma. J Natl Compr Canc Netw. 2012;10:49-57.

3. Zauderer MG, Kass SL, Woo K, Sima CS, Ginsberg MS, Krug LM. Vinorelbine and gemcitabine as second- or third-line therapy for malignant pleural mesothelioma. Lung Cancer. 2014;84:271-274.

4. Zucali PA, Ceresoli GL, Garassino I, et al. Gemcitabine and vinorelbine in pemetrexed-pretreated patients with malignant pleural mesothelioma. Cancer. 2008;112:1555-1561.

5. Alley EW, Lopez J, Santoro A, et al. Clinical safety and activity of pembrolizumab in patients with malignant pleural mesothelioma (KEYNOTE-028): preliminary results from a non-randomised, open-label, phase $1 \mathrm{~b}$ trial. Lancet Oncol. 2017;18:623-630.

6. Armato SG III, Nowak AK. Revised modified response evaluation criteria in solid tumors for assessment of response in malignant pleural mesothelioma (version 1.1). J Thorac Oncol. 2018;13:1012-1021.

7. Wahl RL, Jacene H, Kasamon Y, Lodge MA. From RECIST to PERCIST: evolving considerations for PET response criteria in solid tumors. J Nucl Med. 2009;50(suppl 1):122S-150S.

8. Baas P, Fennell D, Kerr KM, et al. Malignant pleural mesothelioma: ESMO clinical practice guidelines for diagnosis, treatment and follow-up. Ann Oncol. 2015;26(suppl 5):v31-v39.

9. Popat S, Curioni-Fontecedro A, Polydoropoulou V, et al. A multicentre randomized phase III trial comparing pembrolizumab $(\mathrm{P})$ vs single agent chemotherapy (CT) for advanced pre-treated malignant pleural mesothelioma (MPM): results from the European Thoracic Oncology Platform (ETOP 9-15) PROMISE-meso trial. Ann Oncol. 2019;30(suppl 5):v931.

10. Metaxas Y, Rivalland G, Mauti LA, et al. Pembrolizumab as palliative immunotherapy in malignant pleural mesothelioma. J Thorac Oncol. 2018;13:17841791.

11. Lopci E, Zucali PA, Ceresoli GL, et al. Quantitative analyses at baseline and interim PET evaluation for response assessment and outcome definition in patients with malignant pleural mesothelioma. Eur J Nucl Med Mol Imaging. 2015; 42:667-675.

12. Francis RJ, Byrne MJ, van der Schaaf AA, et al. Early prediction of response to chemotherapy and survival in malignant pleural mesothelioma using a novel semiautomated 3-dimensional volume-based analysis of serial ${ }^{18} \mathrm{~F}$-FDG PET scans. J Nucl Med. 2007;48:1449-1458. 